\title{
Bacteriological and pathological studies on avian Mannheimia hemolytica and Pasteurella multocida in Beni- Suef Governorate
}

\author{
Samia I. Afifi ${ }^{1}$, E. A. Mahdy ${ }^{2}$, K. A. El-Nesr ${ }^{2}$ \\ ${ }^{1}$ Animal Health Research Institute, Beni-Suef Branch and ${ }^{2}$ Department of Pathology Faculty of \\ Veterinary Medicine, Beni-Suef University, Beni-Suef 62511, Egypt.
}

\begin{abstract}
Avian Mannheimia hemolytica and Pasteurella multocida were investigated in different poultry farms at Beni-Suef governorate. Bacteriological examination of 160 samples which were taken from organs of freshly dead and diseased chickens revealed isolation of $50(31.25 \%)$ isolates of Pasteurella.Spp., $16(10 \%)$ isolates were Mannheimia hemolytica and 34(21.25\%) isolates were Pasteurella multocida. Experimental infection was carried out on four weeks old Balady chickens which were inoculated with isolates of Mannheimia hemolytica and Pasteurella multocida separately, mortality rate in both reached to $80 \%$. Samples were taken from dead chickens and examined bacteriolgically and histopathologicaly and Pasteurella. Spp. were reisolated from experimentally infected chickens. Antibiogram study with 10 types of chemotherapeutic agents revealed that both microorganisms were sensitive to ceftiofor, gentamycin and lincomycin + spectinomycin. The gross and microscopic pathologic lesions were variable in type and severity in field and experimentally infected cases. There was general hyperemia which most evident in veins of the abdominal viscera. Petecheal haemorrhages were frequently found and widely distributed. Livers of the most acutely affected birds were swollen and had multiple small focal areas of coagulative necrosis and heterophilic infiltration. Heterophilic infiltration also occurs in lungs and certain other parenchymotous organs.
\end{abstract}

Gallibacterium was recently established as a new genus including organisms previously reported as Pasteurella anatis, Actinobacillus salpingitis and avian Pasteurella hemolyticalike organisms (Mannheimia hemolytica), (Bojesen et al., 2007). The new genus Gallibacterium was separated from other genera of family Pasteurellacae according to differences in phenotypic characterization, (Bojesen et al., 2003a; Christensen et al., 2003). Bojesen et al., (2003b) stated that hemolytic Gallibacterium was highly prevalent in birds from production systems with moderate or low level of biosecurity, Christensen et al., (2003) recoded that avian Mannheimia hemolytica caused salpingitis and peritonitis in layer chickens and Bojesen et al., (2007) isolated 75 Gallibacterium field isolates from Mexican chicken egg layers. On the other hand P.multocida, the main causative organism of fowl cholera, a highly contagious disease that affects 100 wild avian species (Botzler 1991). Nakamura et al., ( 1997 ) isolated Pasteurella Spp. from an outbreak of comb necrosis in layer breeder chickens, Sarkozy et al.,(2002) succeeded in inducing experimental fowl cholera in 60 healthy 10 weeks old broiler chickens and Woo and Kim
(2006) isolated P. multocida from two outbreaks of fowl cholera in Korea

The clinical signs of Mannheimia hemolytica were including pneumonia Mwangota et al., (1978), sinusitis, nasal discharge and drop in egg production Lin et al., (2001), and in case of P. multocida it were comb necrosis and lameness Nakamura et al., (1997), orofacial edema, swollen and edematous wattles and combs, and severe respiratory disorders in infected chickens Woo and Kim (2006).

Regarding to necropsy findings due to Mannheimia hemloytica the main lesions were nodular necrosis of the liver Addo and Mohan (1985) peritonitis, Bojesen et al., (2004a) and in case of P. multocida were hyperemia of the trachea, edema of the lungs, increase of pleural fluid and petechial hemorhages in liver, heart and duodenum, minute necrotic foci in the liver and bacterial clumps in the foci Takaya et al., (1981). Liquefactive necrosis of epidermal epithelial cells with hyperplasia, vesicle formation in epidermis, congestion and hemorrhage with fibrinous thrombi in blood vessels of underlying dermis in comb were noted, Nakamura et al., (1997).

The present work was aimed to isolate 
Mannheimia hemolytica and P.multocida from poultry farms with a history of diarrhoea and respiratory disorders, and to study the difference in biological properties between the two mircroorganisms, moreover reporting the clinical signs and the pathological affections and the in vitro sensitivity of the isolated isolates to different chemotherapeutic agents

\section{Materials and methods}

Samples. A total number of 160 freshly dead and diseased chickens $2-8$ weeks old showing diarrhoea, respiratory disorders and mortality rate reached to $20 \%$ were collected from different private farms at Beni-Suef Governorate. The birds were submitted to bacteriological and pathological examinations.

Isolation and identification. Isolation and identification of Pasteurella Spp. was carried out according to (Cruick-Shank et al., 1975; Collee et al., 1996; Christensern et al., 2003).

The internal organs of examined birds (liver, heart, spleen, lungs and trachea as well as nasal and eye discharges ) were inoculated into broth media and incubated for $18-24 \mathrm{~h}$ then strecked into solid media, dextrose starch agar, blood agar, MacConkeys agar and incubated at $37^{\circ} \mathrm{C}$ for $24 \mathrm{~h}$, they were examined for hemolytic or non hemolytic activity. Differentiation of suspected colonies were done through colonial morphology hemolysis, staining reaction, cellular morphology, biochemical reactions specially carbohydrate fermentation, indol production, motility, catalase and oxidase tests.

\section{Experimental infection.}

In chickens. Thirty apparently healthy Balady chikens 4 weeks old were used, it was proved that they were free from both Mannheimia hemolytica and P. multocida through cultural and serological examination, the chickens were divided into 3 groups: group one, ten birds, was used for testing the pathogenicity of Mannheimia hemolytica, group two, ten birds, was used for testing the pathogenicity of P.multocida which was isolated from naturally infected chickens and group three, ten bird, was kept in parallel as uninfected controls. Group one and group two will be inoculated separately intravenous with $0.1 \mathrm{ml}$ of overnight broth containing $3.3 \times 10^{7}$ colony forming units of Mannheimia hemolytica or $P$. multocida according to Lin et al., (2001).

The mortality rate was determined in each group and dead birds were submitted for bacteriological and pathological examinations.
Infection of mice. Fifteen Swiss albino mice 15 $-20 \mathrm{~g}$ were divided into three groups: group one, five mice, was used for determination of the lethal activity of some isolates of Mannheimia hemolytica, group two, five mice, was used for determination of the lethal activity of some isolates of P.multocida and group three, five mice, was used as control, the mice were kept under observation for 2 weeks before they were inoculated. Groups one and two were injected subcutaneously with $0.2 \mathrm{ml}$ of $10^{6}$ C.F.U of Mannheimia hemolytica or $P$. multocida obtained from diseased birds separately .Mice died within 24-48 h after injections were subjected to postmortem examination and reisolation of Pasteurella Spp. was carried out from infected mice Cruick-Shank et al., (1975).

Antibiogram of the isolated Pasteurella Spp. The sensitivity of the isolated Pasteurella Spp. to different antibacterial agents was done by disc diffusion method using dextrose starch agar medium according to Cruick-Shank et al., (1975) the following discs were used, ceftiofur $(30 \mathrm{mg})$, chloramphenicol ( $30 \mathrm{mg}$ ), lincomycin (15mg), and spectinomycin (200mg), amoxicillin (25mg), norfloxacin (10mg), neomycin (30mg), colistin sulphate $(10 \mathrm{mg})$, gentamycin $(30 \mathrm{mg})$, streptomycin $(10 \mathrm{mg})$ and doxycyclin $(30 \mathrm{mg})$.

Histopathology. Tissue specimens were taken from liver, heart, spleen, intestine, brain and lungs of diseased and experimentally infected chickens and fixed in10\% neutral buffered formalin and processed routinely for paraffin embedding techniques. Embedded tissues were sectioned at 4-6 um and stained with hematoxylin and eosin stain according to Bancroft and Stevens (1996).

\section{Results}

Clinical examination. Diseased chickens showed anorexia, depression, ruffled feathers, bilateral or unilateral inflammation of eye and diarrhoea.

Necropsy findings were represented by congestion of the carcasses, necrotic foci and multiple petechae on liver and spleen, congestion and edema of the lungs and hemorrhages all over the intestine.

Bacterial examination. Out of 160 samples of chickens 50 isolates of Pasteurella Spp. were isolated (31.25\%) 16 isolates were Mannheimia hemolytica and 34 isolates were P. multocida. (Table 1)

Regarding the biological properties of isolates of, Mannheimia hemolytica, they were Gram-negative, non-motile, rod-shaped or 
Table (1): Incidence of both Mannheimia hemolytica and P. multocida.

\begin{tabular}{ccccccc}
\hline \multirow{2}{*}{ Number of Samples } & \multicolumn{2}{c}{ Positive Samples } & Mannheimia hemolytica & \multicolumn{2}{c}{ P. multocida } \\
\cline { 2 - 7 } & No. & $\%$ & No. & $\%$ & No. & $\%$ \\
\hline $\mathbf{1 6 0}$ & 50 & 31.25 & 16 & 10 & 34 & 21.25 \\
\hline
\end{tabular}

Table (2): The in vitro sensitivity of the isolates of Mannheimia hemolytica and $P$. multocida to different chemotherapeutic agents.

\begin{tabular}{|c|c|c|c|c|c|c|}
\hline \multirow{3}{*}{ Chemotherapeutic agents } & \multicolumn{3}{|c|}{ Mannheimia hemolytica (16) } & \multicolumn{3}{|c|}{ P. multocida (34) } \\
\hline & \multirow{2}{*}{ Resistant } & \multicolumn{2}{|c|}{ Sensitive } & \multirow{2}{*}{ Resistant } & \multicolumn{2}{|c|}{ Sensitive } \\
\hline & & No. & $\%$ & & No. & $\%$ \\
\hline Ceftiofur & 0 & 16 & 100 & 0 & 34 & 100 \\
\hline Lincomycin+spectinomycin & 4 & 12 & 75 & 9 & 25 & 73.53 \\
\hline Norfloxacin & 11 & 5 & 31.25 & 24 & 10 & 29.41 \\
\hline Chloramphenical & 12 & 4 & 25 & 31 & 3 & 8.82 \\
\hline Amoxicillin & 16 & 0 & 0 & 34 & 0 & 0 \\
\hline Neomycin & 16 & 0 & 0 & 34 & 0 & 0 \\
\hline Colistin Sulphate & 14 & 2 & 12.5 & 29 & 5 & 14.7 \\
\hline Gentamycin & 3 & 13 & 81.2 & 4 & 30 & 88.23 \\
\hline Streptomycin & 14 & 2 & 12.5 & 34 & 0 & 0 \\
\hline Doxycyclin & 11 & 5 & 31.25 & 31 & 3 & 8.82 \\
\hline
\end{tabular}

pleomorphic with cells occurring singly and in pairs, colonies on - bovine blood agar are mostly strongly B-hemolytic, urease, ornithine decarboxylase and Indole negative, oxidase and catalase positive ferment mannose, maltose, glucose, mannitol, glactose and grow on Macconcky agar, P. multocida isolates were Indol, catalase and oxidase positive non hemolytic and ferment mannose, glucose, galactose and mannitol, all injected mice died within $48 \mathrm{~h}$ and showed typical lesions of Pasteurella Spp. In case of experimentally infected chickens the clinical signs appeared 24 to $72 \mathrm{~h}$ post injection, they were off food, lacrimation, fever and emaciation, the mortality rates in both reached to $80 \%$, the post mortem examination showed congestion of lung, liver and spleen, minute necrotic foci on the liver surface and enteritis. The microorganisms were reisolated from organs of infected birds, survived birds were able to overcome the infection, and control birds were negative for bacteriological examination.

The in vitro sensitivity of the isolated isolates of Mannheimia hemolytica and $P$. multocida to different chemotherapeutic agents were indicated in (Table 2).

Pathological examination.

Gross lesions. The gross lesions were variable and appeared to be more severe in field cases. The gross and microscopic lesions induced by Mannheimia hemolytica and those induced by Pasteurella multocida were similar. There was general hyperemia and was most evident in the blood vessels of the abdominal viscera.
Subepicardial and subserosal hemorrhages and also hemorrhages in the lung, abdominal fat and intestinal mucosa were found. The liver of some acutely affected birds was swollen and showed pale necrotic foci.

Microscopic examination. The blood smears, of the experimentally infected birds, stained with Leishman's stain, showed the bipolarity of the microorganisms (Fig.1).

Liver. The examined livers of both field and experimentally infected cases showed multifocal heterophilic infiltrations (Fig. 2) associated with coagulative necrosis in most cases. In subacute cases, the infiltrating neutrophils decreased in number with increased number of the infiltrating mononuclear cells (Fig. 3).

Intestine. In many cases, the intestinal lumens were filled with inflammatory exudate and the submucosa showed heterophilic infiltrations (Fig. 5).

Lungs. The lungs of the affected birds showed heterophilic infiltration of the alveolar walls, peribronchiolar, and intraluminally in the bronchioles (Fig. 7). The lesions in subacute cases, showed mononuclear cell infiltration with giant cell formation (Fig. 8).

Heart. In few cases, there were hemorrhages (Fig. 10) and heterophilic infiltration (Fig. 11) in the myocardial interstitum.

Spleen. The spleens of a few cases showed focal heterophilic infiltrations (Fig. 13) within the splenic parenchyma.

Brain. Microscopically, the examined brains showed no detectable lesions. 

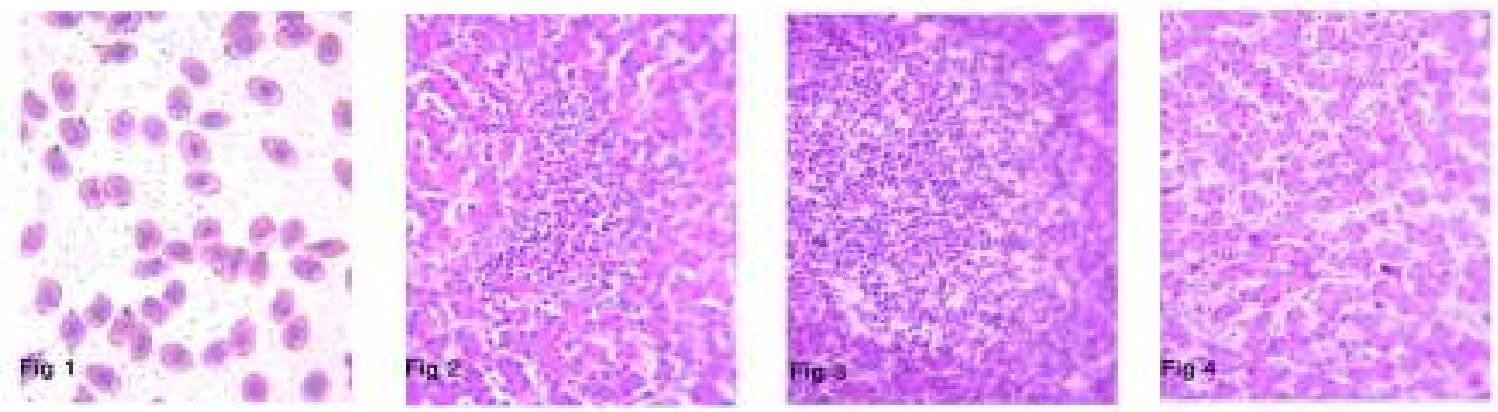

Fig.1:Avian pasteurellosis. Blood smear (notice the bipolarity) Leishman's stain (X1000)

Fig.2:Acute avian pasteurellosis. Heterophilic infiltration in chicken liver ( H\&E X400)

Fig.3: Subacute avian pasteurellosis. Focal mononuclear cell infiltration (H\&E X400)

Fig.4:Chicken liver. Normal histological structure. (H\&E X400)
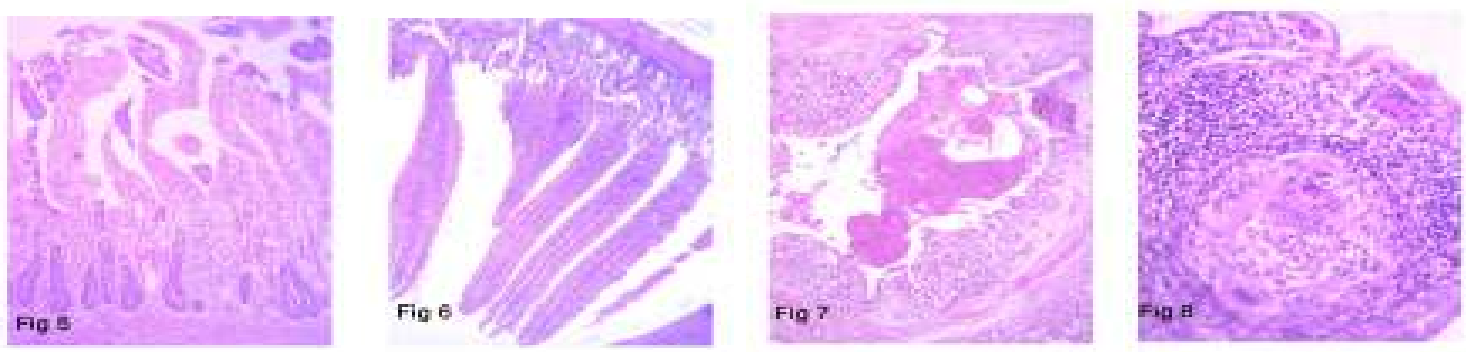

Fig.5: Intestine. Submucosal neutrophilic infiltration. The lumen is filled with inflammatory exudates (H\&E X100)

Fig.6: Intestine. Normal histological structure of intestinal villi and crypts (H\&E X100)

Fig.7: Lung. The bronchiolar lumen is filled with inflammatory inflammatory exudates (H\&E X100)

Fig.8: Lung. Chronic inflammatory reaction with giant cell formation (H\&EX400)
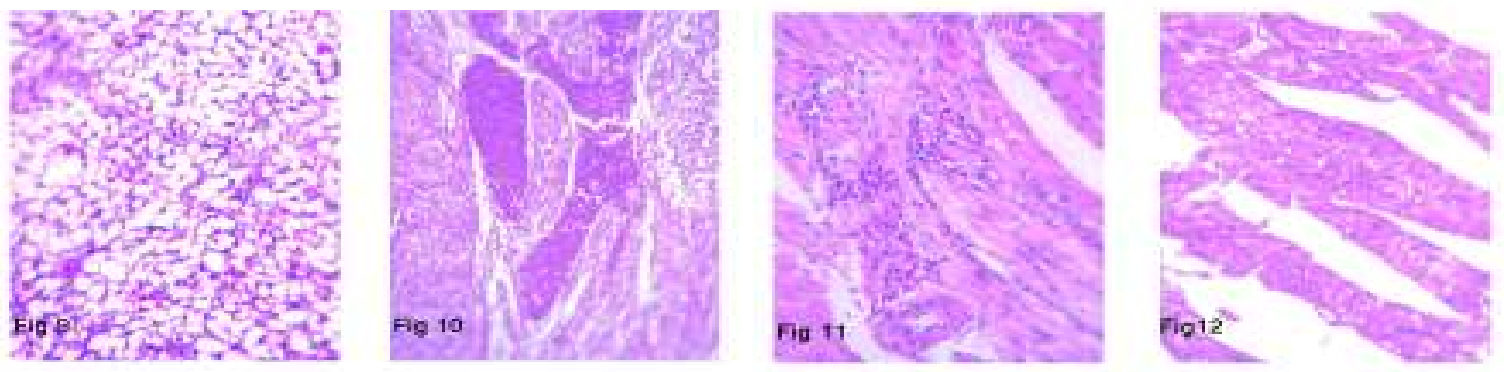

Fig.9: Control lung showed normal pulmonary tissue (H\&E X4UU).

Fig.10: Heart. The myocardial blood vessels showed congestion and hemorrhages (H\&E 200)

Fig.11: Heart. Focal heterophilic infiltrations were in between the myocardial fiber (H\&E X400)

Fig.12: Heart. Normal branched and striated myocardial fibe (H7E X400)
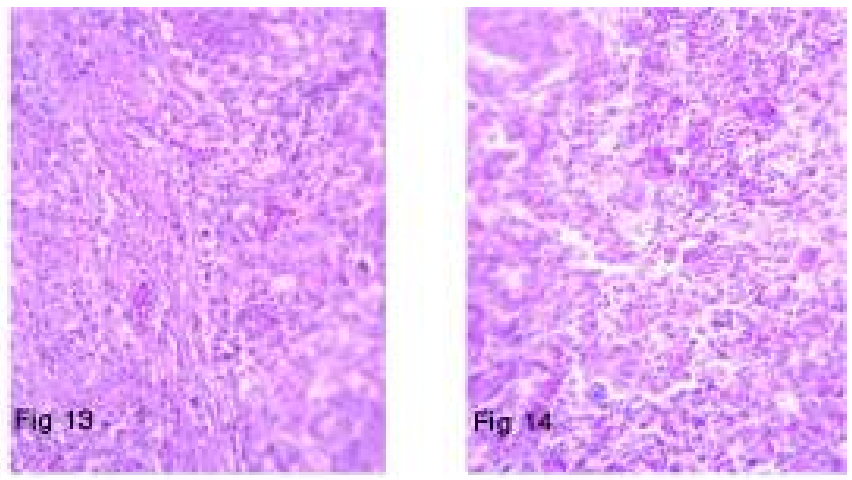

Fig.13: Spleen. Focal heterophilic infiltrations of the splenic parenchyma (H\&E X400)

Fig.14: Spleen. Normal histological structure (H\&E X400) 


\section{Discussion}

Avian Mannheimia hemolytica were classified as a new genus within the family Pasteurellaceae on the basis of unique phenotypic characteristics, they mostly associated with respiratory diseases or septicemia (Christensen et al., 2004; Bojesen el al., 2007).

In the present study, $16(10 \%)$ isolates of Mannheimia hemolytica were isolated from birds showing signs of respiratory affections and mortality rate reached to $(20 \%)$ this nearly resembling the results recorded by Lin et al., (1993), who recorded (30\%) mortality due to Mannheimia hemolytica infection and differ from Show et al., (1990) who recorded higher incidence and increased mortality. In case of P. multocida which revealed from diseased birds with higher percentages $(21.25 \%)$ this agreed with Woo and Kim (2006) who recorded 2 outbreaks of fowl cholera due to P. multocila in korea.

The morphology and biochemical characters of Mannheimia hemolytica and P. multocida were somewhat differed from each others, the organisms were Gram negative, bipolar rodes or coccobacilli, Mannheimia hemolytica were hemolytic, oxidase and catalase positive, indol negative ferment mannose, maltose, mannitole, glucose and galactose, grow well on MacConcky agar, these results agreed with Addo and Mohan (1985); Angen et al., (1999); Christensen et al., (2003); Blackall et al., (2005). P. maltocida differ from Mannheimia hemolytica in that it was non hemolytic on blood agar medium didn't grow on Macconcky agar, and indol positive results agreed with Karaivnov (1984); Collee et al., (1996). Experimental infection of chickens with both of Mannheimia hemolytica and P. multocida caused morbidity and mortality reached to $80 \%$ in both cases this resembling the results recorded by Curtis and Allerhead (1981); Lin et al.,(2001); Bojesen et al., (2004a).

The survived birds didn't harper the infection and this agreed with Woo and Kim (2006); Byarugaba et al.,(2007) and disagreed with Karaivanov (1984) who studied 73 isolates of P. multocida 48 from acute cases of fowl cholera and 25 isolates of chronic fowl cholera. All mice dead within $48 \mathrm{~h}$ post infection and this achieved the results recorded by Yancey et al., (1987); Harper et al., (2003); Chung et al., (2005).

Antibiogram study with 10 types of chemotherapeutic agents revealed that both
Mannheimia hemolytica and P. multocida were $100 \%$ sensitive to ceftiofor , from $81-88 \%$ were sensitive to gentamycin, $73-75 \%$ were sensitive to lincomycin + spectinomycin, from $29 \%$ to $31 \%$ were sensitive to norfloxacin and some isolates of Monnheimia hemolytica were sensitive to chloramphinicol and doxycycline while most isolates were resistant to colistin sulphate and streptomycin and all isolates were resistant to Amoxicillin and streptomycin this some what differ from Semjen et al., (1981) and Sarkozy et al., (2002) who showed that doxycyclin and norfloxacin respectively were highly effective against P. multocida and agreed with Yancey et al., (1987) who recorded that P. multocida and Mannheimia hemolytic from chickens were highly sensitive to cetiofor and Lin et al., (2001) who showed that both Mannheimia hemolytica and P. multocida were sensitive to ceftiofur, amoxicillia, lincomycin and spectionomycin, moderately sensitive to tetracycline, enrofloxacin and slightly sensitive to chloramphenicol.

The pathogenicity or virulence of $P$. multocida in relation to fowl cholera is complex and variable, depending on the strain, host species, and variation within the strain or host and conditions of contact between the two. The ability of P. multocida to invade and reproduce in the host is enhanced by the presence of a capsule that surrounds the the organism (Saif et al., 2003).

Endotoxins are produced by all $P$. multocida isolates, both virulent and non virulent. They may contribute to virulence; however invasion and multiplication of a strain are necessary for the production of sufficient quantities of endotoxins in vivo to contribute the pathological processes (Saif et al., 2003).

The gross lesions and clinical signs including septicemia, respiratory disorders and diarrhoea resembling the signs which were recorded by Mwangota et al., (1978); Nakamara et al., (1997); Lin et al., (2001); Bojesen et al., (2004 b); Woo and Kim (2006).

Regarding to necropsy findings due to Mannheimia hemloytica the main lesions were nodular necrosis of the liver Addo and Mohan (1985) peritonitis, Bojesen et al., (2004a) and in case of $P$. multocida were hyperemia of the trachea, edema of the lungs, increase of pleural fluid and petechial hemorhages in liver, heart and duodenum, minute necrotic foci in the liver and bacterial clumps in the foci Takaya et al., (1981). Liquefactive necrosis of epidermal 
epithelial cells with hyperplasia, vesicle formation in epidermis, congestion and hemorrhage with fibrinous thrombi of under lying dermis in comb were noted, Nakamura et al., (1997). The gross and microscopic pathology caused by an atypical strain of Pasteurella gallinarum (Frenso strain) was compared in chickens with that caused by the AmericanType Culture Collection type strain. Micrscopically, there was severe chronic pyogranulomatous airsaculitis, pericarditis, perihepatitis, myositis, synovitis, and granulomatous pneumonia. One bird had severe acute multifocal hepatitis Shivaprasad and Droual, (2002).

In our material, the gross and microscopic lesions induced by Mannheimia hemolytica and those induced by Pasteurella multocida were similar. There was general hyperemia and was most evident in the blood vessels of the abdominal viscera. Subepicardial and subserosal hemorrhages and also hemorrhages in the lung, abdominal fat and intestinal mucosa were found. The liver of some acutely affected birds was swollen and showed pale necrotic foci. Microscopically, there were heterophilic infiltrations involving most of the parenchymatous organs. Stress factors (e.g. massive population)

Play an important role in aggravated and increased severity of the pathological lesions in case of field cases in comparison with those in case of experimentally infected birds.

Recruitment of heterophils into the respiratory tract during infection was found to contribute considerably to the lung lesions in chickens and was found to mediate tissue damage, possibly allowing a more rapid systemic spread of P. multocida. However, during progression of the infection, the heterophil mediated-necrosis in chickens seemed to stimulate giant cell demarcation of infected lung tissue, which coincided with the clearance of $P$. multocida from the spleen, thus harpering further invasion. Consequently, heterophil activation plays a dual role for the outcome of P. multocida infection in chickens, where it initially seems to promote invasion and systemic spread but subsequently helps in limit the infection by giant cell formation and bacterial clearance Bojesen et al., (2004b).

Chronic localized infections can involve the middle ear and cranial bones and have been reported to result in torticollis in turkeys. Heterophilic infiltration and fibrin were observed in the air spaces, middle ear, and meninges. Multinucleated giant cells were often associated with necrotic masses of heterophils in air spaces Saif et al., (2003).In conclusion, bacteriological examination of 160 samples of freashly dead and diseased chickens suffering from respiratory disorders and diarrhea obtained from private farms at Beni Suef Governorate revealed isolation of $16(10 \%)$ isolates of Mannheimia hemolytica and 34(21.25\%) isolates of $P$. multocida. Experimental infection with isolated isolates of both microorganisms separately in 4 weeks old chickens produced the same characteristics lesions of Mannheimia hemolytica and $P$. multocida within 3 days post infection. The in vitro sensitivity tests have shown that both Mannheimia hemolytica and P. multocida were highly sensitive to ceftiofur, gentamycin and lincomycin and spectinomycin. The present study has cleared the occurrence of Mannheimia hemolytica and P. multocida in poultry farms in Beni Suef Governorate and their sensitivity for some chemotherapeutic agents.

\section{References}

Addo P. B. and Mohan K. (1985): A typical P. hemolytica type A from Poultry. Avian Dis. 29 (1): $214-217$

Angen-O.; Mutters R.; Cougant D. A.; Olsen J. E. and Bisgaard M. (1999): Taxonomic relationships of the $P$. hemolytica complex as evaluated by DNA - DNA hybridization and 16S rRNA sequencing with proposal of Mannheimia hemolytica gen. nov., comb. nov., Mannnheimia granulomatis comb. nov., Monnheimia glucosida SP. nov., Mannheimia ruminalis - SP. nov. and mannheimia varigena SP. nov., Int. Sys. Bac. Jan; 49 Ptl: 67 $-86$.

Bancroft, D. and Stevens, A. (1996): Theory and Practice of histological techniques. $2^{\text {nd }}$ ed. Churchill Livingstone,Edinburgh,London,Melboure

Blackall P. J.; Christensen H.; Beckenham T. Blackall L. L.; and Bisgaard M. (2005): Reclassification of P. gallinarum, Hemophilus paragallinarum, P. Avium and P. Volantium as Avibacterium gallinarum gen. nov, comb. nov., Avibacterium Paragallinarum - comb. nov.,Avibacterium avium comb. nov., and Avibacterium Volantium - Comb. nov. -Int Syst Evol. Mic. Jan; 55 (Ptl): $353-62$.

Bojesen A. M.; Christensen H.; Nielsen O. L.; Olsen J. E.; and Bisgaard M. (2003a): Detection of Gallibacterium Spp. in Chickens by Fluorescent $16 \mathrm{~S}$ rRNA in situ hybridization. J. Clin. Microbiol. 41 (11): 5167 - 72.

Bojesen A. M.; Nielsen S. S. and Bisgaard M. (2003b): Prevalence and transmission of hemolytic Gallibacterium species in chicken production systems with - different biosecurity levels. Avian Pathol., 32 (5): 503 - 510.

Bojesen A. M.; Nielson O. L.; Christensen J. P. and Bisgaard M. (2004a): In vivo studies of Gallibacterium anatis infection in chickens. Avian Pathol., 33(2): 145152.

Bojesen A. M.; Petersen K. D.; Nielson O. L.; Christensen J. P. and Bisgaard M. (2004b): Pasteurella multocida infection in heterophil - depleted chickens. Avian Dis. Sep; 48 (3): 463 - 70. 
Bojesen - A. M.; Vazquez M. E.; Robles, F.; Gonzalez C.; Soriano E. V.; Olsen J. E. and Christensen H. (2007): Specific identification of Gallibacterium by a P.C.R using Primers targeting the $16 \mathrm{~s} \mathrm{r}$ RNA and $23 \mathrm{~S} \mathrm{r}$ RNA genes.Vet. Microbiol., 20; 123 (1-3): 262 - 268.

Botzler, R.G. (1991): Avian Cholera on orth coast California, distinctive epizootiological features Ann Ny. Acad. Sci., 969: 224 - 228.

Byarugba D. K.; Minga, U. M.; Gwakisa P. S.; Katunguk, R.; Wakishaya, E.; Bisgaard M. and Olsen J. E. (2007): Virulence characterization of Avibacterium paragallinarum strains from Uganda. Avian Pathol ., 36 (1): 35-42.

Christensen, H.; Bisgaard, M.; Aalbaek, B. and Olsen J. E. (2004): Reclassification of Bisgaard taxon 33, with Proposal of volucribacter psittacicida gen. nov., sp. nov., and volucribacter amazonee SP. nov. new members of The pasteurellaceae. Int. J. Syst. Evol. Microbiol., 54: 813 818.

Christensen, H.; Bisgaard, M.; BoJesen, A. M.; Matters, R. and Olsen J. E. (2003): Genetic relationships among avian isolates classified as $P$. haemolytica, Actinobcillus salpingitidis or $P$. anatis with proposal of Gallibacterium anatis gen. nov. comb. nov and description of additional genomospecies within Gallibacterium gen. nov. Int. J Syst. Evol. Microbiol., 53(Pt): 275- 287.

Chung, J. Y.; Wilkie, I.; Boyce, J. D. and Adler B. (2005): Vaccination against fowl cholera with acapsular P. multocid A:1.Vaccine, 23 (21): 2751 - 2755.

Collee, J. G.; Fraser, A. C.; Marmion, B. P. and Simmons, A. (1996): Practical medical microbiology. $14^{\text {th }}$ Ed. Charchill Livingstone.New York, Edinbourgh London, Madrid Melbourne, Sanfrancisco and Tokyo.

Cruick-Shank, R.; Duguid, J. P.; Marion, B. P. and Swain, R. H. A. (1975): Medical Microbiology. $12^{\text {th }}$ ed., Vol II. Churchill Livingstone, Edinburgh, London and New-York.

Curtis P. E., and Allerhead G. E. (1981): Investigation to determine whether healthy chickens and turkeys are oral carriers of pasteurella multocida. Vet. Rec., 108 (10): 206 207.

Harper, M.; Boyce, J. D.; Wilkie, I. W. and Aler, B. (2003): Signature-tagged mutagenesis of P. multocida identifies mutants displaying differential virulence characteristics in mice and chickens. Infec. Immmunol., 71(9): $5440-5446$.

Karaivanov L. (1984): Biochemical tests for identifying pasteurella multocida. Vet. Med. Nauki, 21 (9): $38-44$.

Lin, M. Y.; Cheng, M.C.; Huang, K. J. and Tsai, W.C. (1993): Classification, Pathogenicity and drug susceptibility of hemolytic gram - negative bacteria isolated from sick or dead chickens. Avian Dis., 37 (1): 6 - 9.

Lin, M. Y.; Lin, K. J.; Lan, Y. C.; Liaw, M. F. and Tung M. C. (2001): Pathogenicity and drug susceptibility of the Pasteurella anatis isolated in chickens in Taiwan. Avian Dis., 45 (3): 655 -658.

Mwangota, A. U.; Muhammed S. I. and Thomson R.G. (1978): Serological types of $P$. hemolytica in - Kenya. Cornell Vet. Jan; 68 (1): 48 - 93.

Nakamura, K.; Shirai, J.; Imai, K.; Hihara H., and Tanimuro, N. (1997): Outbreak of comb necrosis in breeder chickens. Avian Dis., 41 (1): 252 - 256.

Saif, Y. M.; Barnes, H. J.; Glisson, J. R.; Fadly, A. M.; Mcbougald, L. R. and Swayne, D. E. (2003):Diseases of poultry. $11^{\text {th }}$ ed. Iowa State Press.a Blaclwell Publishing Company.

Sarkozy, G.; Semjen, G.; laczay, P. and Horrath, E. (2002): Treatment of experimentally induced P. multocid infections in broilers and turkeys comparative studies of different oral treatment regimens. J-Vet. Med. B Infect. Dis. Vet Puplic Health, 49 (3): 130 -134.

Semjen, G.; Magyar, T. and Laczay, P. (1998): Therapeutic effecancy of doxycycline against experimental P. multocida infection in broiler chickens. Acta Vet. Hung 46 (1): $85-93$.

Shaw D.P., Cook D.B. Maheswaran S.K., Lindeman C.J., and Holvorson D.A (1990): $P$. hemolytica as a CoPathogen in Pullets and laying hens. Avian Dis., 34(4): $1005-1008$.

Shivaprasad, H. L and Droual, R. (2002): Pathology of an atypical strain of Pasteurella gallinarum infection chickens. Avian Pathol., 31(4): 399-406.

Takaya, M.; Akiyama, K .; Taniguchi, T.; Nonomura, I. and Horiuchi T.(1981): Fowl cholera found in imported myna birds (Eulabes intermedia ) Nat. Inst. Animal Health, Q (Tokyo ).fall, 21 (3) : 129 -33.

Woo Y.K. and Kim J.H. 2006: Fowl Cholera outbreak in domestic poultry and epidemiological properties of $\mathrm{P}$. maltocida isolate. J. Microbiol., 44 (3): 344 - 353.

Yancey, R. J.; Kinney, M. L.; Roberts, B. J.; Good enough, K. R.; Hamel, J. C. and Ford, C. W. (1987): Ceftiofur Sodium, a broad-spectrum cephalosporin, evaluation in vitro and in vivo in mice. Am. J. Vet. Res., 48 (7): $1050-1053$.

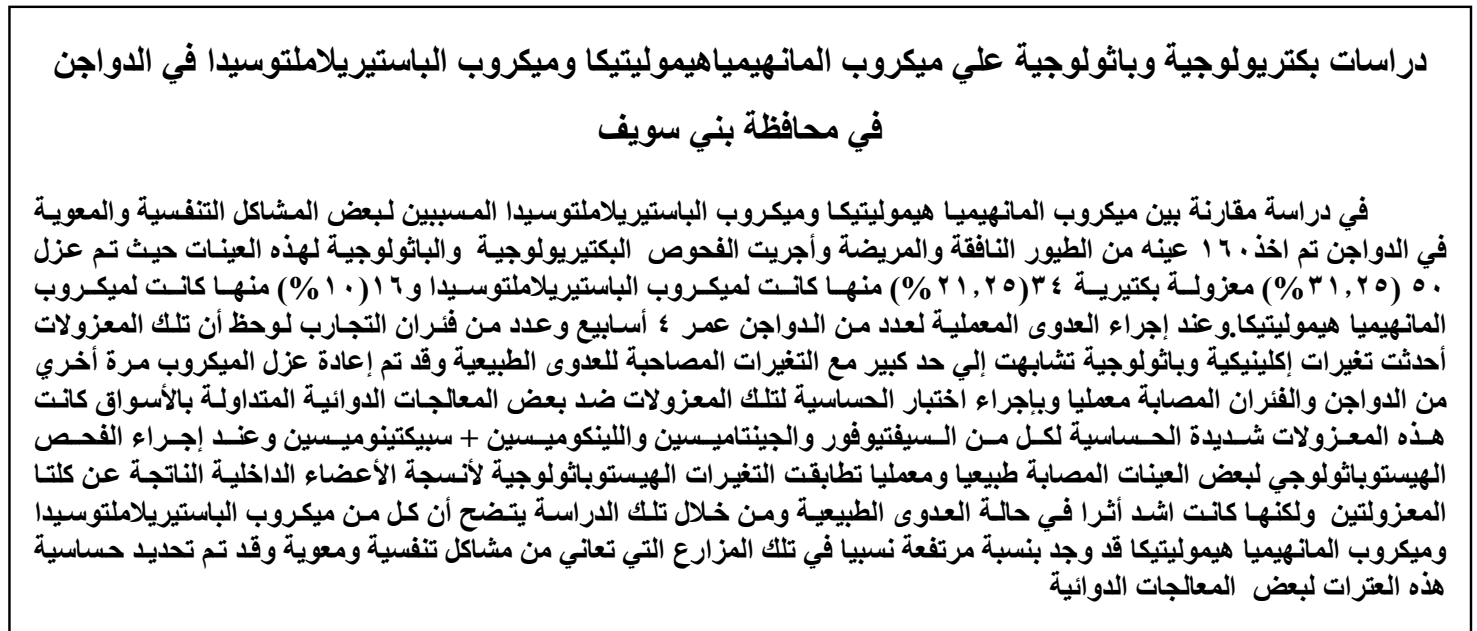


